\title{
Mejora de los procesos de lectura a través del Programa MATEL
}

Rosa M. Molina Roldán. Equipos de Orientación Educativa Francisco Pérez Segura. Orientador de Educación Secundaria Adela M. Molina Roldán. Maestra de Educación Primaria

Almería, España

frapese@supercable.es 


\section{RESUMEN}

Introducción. En la presente investigación pretendimos estudiar la relación existente entre la comprensión, la velocidad y la eficacia lectora. El hecho que motivó la investigación, fue la constatación de la relación existente entre déficit de lectura y fracaso escolar, en un grupo de alumnos de Educación Secundaria Obligatoria pertenecientes a un Colegio Público Rural (Almería, España).

Método. Ante tal situación, un grupo de profesores del centro junto al Equipo de Orientación Educativa, decidimos poner en marcha un proceso de investigación-acción, a través del cual aplicamos y evaluamos el Programa MATEL, cuya propuesta es conseguir eficacia lectora en los alumnos implicados a través de la práctica de una serie de estrategias que conducen a la mejora de la comprensión y la velocidad lectoras.

Resultados. Comprobamos que la comprensión y la velocidad están asociadas a la eficacia lectora, no produciéndose tal asociación entre velocidad y comprensión lectoras. Las diferencias de medias entre las mediciones efectuadas constatan un efecto del tratamiento en la variabilidad de los resultados (velocidad, comprensión y efectividad).

Discusión. Los resultados mostraron, tras la aplicación del Programa durante un curso escolar, que la instrucción fue efectiva de manera diferencial, ya que por una parte los alumnos consiguieron mejorar significativamente su eficacia lectora, y por otra, aumentar también significativamente su velocidad lectora. En el caso de la comprensión lectora, aunque se produjo un aumento significativo en la mayoría de los momentos de la investigación, bajó en el momento final.

PALABRAS-CLAVE: dificultades en la lectura, programas de intervención, procesos de lectura, eficacia lectora. 


\section{INTRODUCCIÓN}

En una sociedad en la que el conocimiento nos rodea y su producción es cada vez mayor, se hace necesario procesar grandes cantidades de información, rápidamente y con efectividad. La lectura eficiente es la gran herramienta para hacerlo. De una lectura eficiente se deriva una escritura eficiente y, por ende, un aprendizaje también eficiente. La lectura para los estudiantes es el principal instrumento de aprendizaje, pues la mayoría de las actividades escolares se basan en ella. Leer es uno de los mecanismos más complejos a los que puede llegar una persona ya que implica decodificar un sistema de señales y símbolos abstractos.

Sin embargo, en nuestra práctica profesional venimos constatando, desde hace algún tiempo, que la lectura es un factor importante a tener en cuenta a la hora de explicar el fracaso escolar. Más específicamente, estamos asistiendo a un déficit de lectura que presentan los alumnos, en particular, y la población, en general. Gran parte del fracaso escolar se debe a que casi siempre se enseña el mecanismo de descifrar sonidos escritos, pero en ocasiones reducidas se llega a ser un buen lector (comprender, obtener una información válida y utilizable, etc).

Hasta hace poco tiempo uno de los factores que explicaban esta deficiente lectura, entre los profesionales de la educación, era la eterna discusión sobre los métodos utilizados para el aprendizaje, bien global o sintético. Actualmente este tipo de discusión está superado, tanto uno como otro ofrecen aportaciones significativas, conservándose del método global "la importancia de la lectura real desde las primeras palabras aprendidas globalmente y la necesidad de conceder un lugar importante a los aspectos funcionales y afectivos del aprendizaje" (Foucambert, 1989, p.15) y de los métodos sintéticos "la presentación sistemática y rigurosa de los elementos inferiores a la palabra y de los diversos componentes de la lengua escrita" (Foucambret,1989, p. 15).

Desde nuestro punto de vista, el planteamiento excluyente de método analítico o método global es erróneo, puesto que aunque se habla de método de aprendizaje se constituyen en realidad como métodos de enseñanza. Ciertamente existe una estrecha relación entre enseñanza y aprendizaje, pero sin embargo lo que realmente interesa es saber de qué manera ha ido construyendo el aprendizaje el propio individuo, y es en este punto en donde la diversidad del alumnado individualiza sus propios niveles de consecución. En cualquier caso, en el 
desarrollo del proceso lector, todos los alumnos, deben poner en juego una serie de procesos y estrategias cognitivas que estarán relacionadas con la finalidad para la que se lee. El uso, más o menos, adecuado de las mismas, como capacidad de autorregulación, son claves en el proceso. La adquisición o no de estas estrategias, está ligada también con la motivación del sujeto y el hábito lector del mismo, entre otras variables.

Es por ello, que, cuando abordamos la investigación, nos propusimos los siguientes objetivos:

1) Analizar en qué medida el aumento de la velocidad influye en la eficacia lectora.

2) Investigar la relación existente entre comprensión lectora y eficacia.

3) Analizar la influencia que la autorregulación conjunta de los factores velocidad y comprensión tienen sobre la eficacia lectora.

\section{MÉTODO.}

\section{Sujetos.}

La muestra de alumnos estuvo formada por un total de 28 alumnos de $1^{\circ}$ ciclo de Educación Secundaria Obligatoria de un CPR (Colegio Público Rural), con edades comprendidas entre 12 y 14 años. Este CPR tiene unidades educativas repartidas por los pueblos cercanos de la comarca. Por ello, la población escolar está formada por alumnos y alumnas de ambos pueblos y de los niveles educativos de Educación Infantil, Educación Primaria y sólo el primer ciclo de Educación Secundaria Obligatoria. El nivel sociocultural de los alumnos es medio-bajo y bajo. Sólo un 38\% de la población estudiada posee algún tipo de estudios (primario hasta universitario), En la Tabla 1 se exponen los porcentajes del nivel cultural de las familias.

Tabla 1. Nivel cultural de las familias de los alumnos

\begin{tabular}{|l|l|}
\hline \multicolumn{1}{|c|}{ NIVEL DE ESTUDIOS } & PORCENTAJE \\
\hline No sabe leer ni escribir & $3 \%$ \\
\hline Sin estudios & $59 \%$ \\
\hline Estudios primarios & $16 \%$ \\
\hline Graduado escolar $^{\prime}$ & $13 \%$ \\
\hline Formación Profesional de $1^{\circ}$ Grado & $4 \%$ \\
\hline Formación Profesional $2^{\circ}$ Grado & $1 \%$ \\
\hline Bachillerato & $1 \%$ \\
\hline Diplomados universitarios & $2 \%$ \\
\hline Licenciados universitarios & $1 \%$ \\
\hline
\end{tabular}




\section{Materiales}

El Programa MATEL utilizado no pretende ser la solución exclusiva para alumnos con problemas sino, más bien, una acción psicopedagógica dirigida al conjunto de la clase. $\mathrm{Su}$ finalidad última consiste en facilitar un aprendizaje lector que permita a cada uno, individualmente, darse cuenta de su propia forma de leer y a partir de este auto-convencimiento ayudarle a que la modifique, la corrija o la mejore. "La integración en el campo competencial del alumno de las estrategias lectoras le permitirá avanzar hacia la autorregulación de su propia actividad lectora" (Serra, y Oller, 1997, p. 24).

Este programa plantea unos objetivos generales, unos objetivos específicos mensurables y otros objetivos específicos no mensurables.

Objetivos generales:

a. Mejorar cualitativamente el saber leer del alumno haciéndole pasar de comportamientos alfabéticos a comportamientos ideo-visuales eficaces.

b. Eliminar las consecuencias de un aprendizaje inicial de la lectura de tipo alfabético que prima la vertiente oral del texto en detrimento de los procesos cognitivos que constituyen el verdadero acto lector. Ayudarle en la ruptura del hábito lector que este cambio puede significar en muchos alumnos.

c. Ayudar al alumno a modificar su actitud pasiva en la lectura y sus expectativas ante el escrito.

Objetivos específicos mensurables.

a. Conseguir con los alumnos y alumnas de $1^{\circ}$ Ciclo de Educación Secundaria Obligatoria una velocidad de lectura superior a 200 palabras por minuto.

b. Conseguir con dichos alumnos y alumnas una comprensión lectora superior al $70 \%$.

c. Obtener un buen índice de eficacia lectora.

Objetivos específicos no mesurables.

a. Ampliar el campo visual en cada fijación.

b. Disminuir el tiempo empleado en cada fijación. 
c. Disminuir el número de regresiones oculares.

e. Potenciar la comprensión del texto.

f. Practicar la anticipación lectora y la hipótesis de sentido.

g. Practicar la utilización de cualquier tipo de texto.

Los ejercicios del Programa se presentan estructurados en 9 series, cada una de las cuales pretende conseguir unos objetivos muy concretos, como son:

Serie 0: pretende desarrollar movilidad y agilidad oculares. Precalentamiento visual y desarrollo de la atención.

Serie A: pretende ampliar el campo visual en cada fijación. Ejercita la capacidad de discriminación visual e identificación. Entrenar la memoria inmediata y elegir estrategias personales para buscar palabras.

Serie B: pretende aumentar el campo visual útil en cada fijación y dificultar la realización de regresiones oculares.

Serie C: pretende mejorar la discriminación visual.

Serie D: pretende desarrollar la rapidez de exploración de un texto utilizando las estrategias convenientes.

Serie E: pretende reforzar los comportamientos anticipatorios para prever mejor la información posterior.

Serie F: pretende evaluar la eficacia del comportamiento lector a partir de los parámetros de velocidad y comprensión.

Serie H: pretende facilitar en el alumno su autoconocimiento del proceso lector en la lectura oralizada . Interiorizar los principios siguientes:

1. La lectura en voz alta presupone una anticipación activa y constante del texto. Si no existe esta anticipación, no hay lectura sino oralización mecánica.

2. Leer en voz alta es leer para otros. Es un acto de comunicación y por tanto es necesario dar una entonación adecuada y expresiva lo que significa que se hace una lectura ideovisual

Los elementos esenciales para evaluar el progreso de alumnos y alumnas durante la aplicación del programa se realiza a través de la SERIE F. 


\section{Procedimiento de investigación-acción.}

Todo el proceso investigador partió de que un grupo de maestros y maestras del Colegio Público Rural, tras evaluar y analizar el nivel de competencia curricular de sus alumnos constataron que, gran parte del fracaso escolar que presentaban, se debía a una pésima eficacia lectora. Así nuestra investigación tuvo las siguientes fases:

\section{1) Planteamiento del problema.}

Este grupo de maestros y maestras pretendían mejorar la eficacia lectora en sus alumnos, convencidos de que, a partir de la consecución de este objetivo, caerían en cascada otros del tipo de: éxito escolar, gusto por la lectura y por tanto hábito lector e interés por descubrir nuevos conocimientos y experiencias..

Nos planteamos la pregunta ¿qué es la lectura? . De acuerdo con los modelos teóricos y conceptuales actuales (Sánchez, E.,1994, p.131), entendemos que es la obtención de informaciones significativas y lo que puede variar de una situación a otra es lo que se quiere hacer con esas informaciones: disfrutar, meditar, informar, jugar, etc. "Los objetivos o intenciones de lectura son, pues, los que deben marcar el qué leer y también el cómo leer" (Grau,1997, p. 34). Así, la lectura se inicia mucho antes que el acto lector, en el momento en que estamos seleccionando un texto ya estamos leyendo ya que, incluso, el propio proceso selectivo ya nos está aportando informaciones. Por tanto, podemos afirmar que aprender a leer es aprender a explorar un texto, unas veces de forma rápida y otra lentamente dependiendo de la intencionalidad que tengamos respecto a ese texto.

La intención del lector, cuando realiza la lectura, influye en su captación. Así, en la misma actividad realizamos tareas diferentes: si leemos para encontrar una información específica, si leemos para captar la idea general, si leemos en profundidad para conocer un texto específico o si leemos para establecer un juicio crítico. Un paso más sería amar la lectura y esto es crear la necesidad de recurrir a un entorno escrito bajo cualquier forma: carteles, anuncios, recetas de cocina, modos de empleo, consignas, poemas, periódicos, etc.

\section{2) Identificación del problema}

Las preguntas planteadas fueron: ¿ qué es lectura eficaz?. ¿de qué depende una buena lectura?. De forma consecuente con la investigación actual (Grau, 1997, p. 32; Serrejón, 1995, pp. 46-47 y Solé, 1992), un lector eficaz es el que lee rápidamente, comprende lo que 
lee, adapta la velocidad y la comprensión lectoras al tipo de texto que lee y a la intención o proyecto con el que lee y recuerda bien lo leído.

Los maestros implicados en la experiencia creían que, algunos aspectos fundamentales para llegar a la eficacia lectora podían ser la comprensión y la velocidad en la lectura, ya que por una parte un buen lector lee de prisa y comprende bien y, por otra, cuanto más rápidamente se lee, más y mejor se comprende.

Comenzamos, pues, a buscar entre la bibliografía referida al tema materiales o programas que se adaptaran tanto a los objetivos que pretendían conseguir como a la disponibilidad de recursos que tenían. Así llegó a sus manos, a través del Equipo de Orientación Educativa el PROGRAMA DE LECTURIZACIÓN MATEL (López Rubio, 1992). Este Programa basa su teoría y práctica en la idea de que para conseguir eficacia lectora es necesario alcanzar una buena velocidad y comprensión respecto a la lectura, con lo cual era justo lo que pretendían los maestros de este centro rural.

De acuerdo con el Modelo de Lecturización MATEL (López Rubio, 1992), para aumentar la velocidad lectora tenemos que partir de los fundamentos de lo que es leer. Al leer nuestros ojos van moviéndose a base de saltos o fijaciones en las que se agrupan una o varias palabras. Por lo tanto, para aumentar la velocidad lo que tenemos que pretender es realizar el menor número de fijaciones posibles en cada línea para así en una sola fijación abarcar el mayor número de palabras posibles, favorecer la interpretación de bloques de palabras en forma instantánea, fortalecer la concentración del lector por medio de la rápida asimilación del texto y de la disminución de los lapsos de tiempo ocioso y ampliar la capacidad de aprendizaje de textos mediante el uso de la técnica de lectura por bloques. Por otra parte, unido estrechamente con lo anterior, para mejorar la comprensión lectora es fundamental una buena exploración que consiste en comprender de que va el texto, antes de ponernos a trabajar en él y una buena lectura que debe ser con el ritmo propio de cada uno, haciendo una lectura general y buscando el significado de lo que se lee.

Velocidad y compresión lectoras no son dos aspectos independientes del proceso lector, están tan íntimamente relacionados que está demostrado que con el incremento de la velocidad lectora se mejora cuantitativa y cualitativamente la comprensión en la lectura. 


\section{3) Planteamiento de hipótesis de intervención}

A partir del modelo previo, las hipótesis de trabajo planteadas fueron las siguientes:

1. La velocidad y la comprensión lectora se perfeccionarán con el entrenamiento de habilidades lectoras del tipo de: incremento del campo visual, discriminación visual y rapidez en la exploración de un texto.

2. La aplicación del Programa MATEL contribuirá a aumentar la eficacia lectora en los alumnos como producto de la mejora en la velocidad y la comprensión.

\section{4) Intervención}

La experimentación se desarrolló durante el $2^{\circ}$ y $3^{\circ}$ trimestre del curso 96/97. Aunque fue un proyecto apoyado por la totalidad del equipo de profesores de $1^{\mathrm{o}}$ ciclo de ESO, donde más incidencia tuvo fue en las área de lengua castellana y literatura y lengua extranjera (inglés). El tiempo total invertido en el desarrollo del Programa fueron unas 60 horas, aproximadamente, repartidas a lo largo de 7 meses de trabajo, con una media semanal de tres horas, repartidas en las siguientes sesiones:

1. Sesiones de entrenamiento individual. A través de ellas se trabajaron las series A, B, C, D, E, G y H. Fueron desarrolladas dentro de la propia aula de los alumnos y de forma autónoma por parte de cada uno de ellos. Aunque todos realizaron el mismo ejercicio, cada uno invirtió el tiempo que estima necesario, controlado por el profesor y anotado por cada uno de los propios alumnos. Se realizaron cuatro sesiones semanales, coincidiendo con el horario del área de lengua castellana, con una duración aproximadamente de 1/2 hora por sesión y distribuidas de la siguiente forma:

Tabla 2. Distribución semanal de las series del programa.

\begin{tabular}{|l|l|l|l|}
\hline \multicolumn{2}{|c|}{ Semanas $1 / 3 / 5 / 7$} & \multicolumn{2}{c|}{ Semanas 2/4/6/8 } \\
\hline Lunes & Serie O & Lunes & Serie C \\
Martes & Serie A & Martes & Serie D \\
Miércoles & Serie F & Miércoles & Serie F \\
Jueves & Serie B & Jueves & Serie E \\
Viernes & Serie H/J & Viernes & Reflexión \\
\hline
\end{tabular}


2. Sesiones de evaluación. Aunque las sesiones de evaluación se realizaron todas las semanas (los miércoles), con una duración aproximadamente de $1 / 2$ hora, a través de las series F, existieron tres momentos o controles a lo largo de todo el proceso que fueron los indicadores que posteriormente utilizamos para la cuantificación de los resultados. Se desarrollaron también en el propio aula, dentro del horario del área de Inglés. La persona encargada de realizar la sesión de evaluación fue la profesional implicada del EOE. La distribución de las sesiones de evaluación a lo largo del desarrollo del Programa, fue la siguiente:

Tabla 3. Distribución de las sesiones de evaluación

\begin{tabular}{||c|c|c|l||}
\hline FECHA & PRUEBA & CURSO & CONTROL \\
\hline \hline $4-12-96$ & F.4 & $1^{\circ}$ ESO & \\
\hline $11-12-96$ & F.1 & $1^{\circ}$ ESO & $1^{\circ}$ Control \\
\hline $8-1-97$ & F.6 & $1^{\circ}$ ESO & \\
\hline $22-1-97$ & F.7 & $2^{\circ}$ ESO & \\
\hline $5-2-97$ & F.4 & $2^{\circ}$ ESO & \\
\hline $19-2-97$ & F.5 & $1^{\circ}$ ESO & \\
\hline $5-3-97$ & F.8 & $2^{\circ}$ ESO & \\
\hline $19-3-97$ & F.2 & $2^{\circ}$ ESO & $2^{\circ}$ Control \\
\hline $9-4-97$ & F.6 & $2^{\circ}$ ESO & \\
\hline $23-4-97$ & F.9 & $2^{\circ}$ ESO & \\
\hline $7-5-97$ & F.7 & $1^{\circ} \mathrm{ESO}$ & \\
\hline $21-5-97$ & F.2 & $1^{\circ}$ ESO & \\
\hline $4-6-97$ & F.3 & $2^{\circ}$ ESO & $3^{\circ}$ Control \\
\hline \hline
\end{tabular}

3. Sesiones de reflexión compartidas. Las realizamos quincenalmente en el salón de usos múltiples. En ellas participaban todos los implicados en la aplicación del Programa: los alumnos, equipo de profesores y profesional del EOE. A través de estas sesiones de reflexión se pretendió analizar la puesta en marcha del Programa, haciendo hincapié en los aspectos positivos y negativos detectados, así como propuestas de mejora. También realizamos el análisis de los resultados y, lo más importante, intentamos que los alumnos y profesores descubrieran cómo la mejora en la eficacia lectora repercute en su proceso de enseñanzaaprendizaje. 
4. Sesiones con padres. Se realizaron también en el salón de usos múltiples, de forma quincenal, y con una duración aproximada de dos horas. En ellas participaron los padres de los alumnos implicados, el equipo de profesores y la profesional del EOE implicada. A través de las sesiones con padres se pretendió conseguir una sensibilización hacia la lectura, a nivel familiar.

\section{5) Evaluación y análisis de resultados}

La evaluación se realizó en las siguientes fases:

1. Evaluación inicial. Los maestros (con los alumnos a los que se les iba a aplicar el programa) evaluaron a los alumnos según las pruebas presentadas en la serie $\mathrm{F}$ al efecto (Texto de Prueba o ensayo y TEST DE CONTROL INICIAL).

2.Evaluación de proceso. Pretende detectar dificultades, objetivos de difícil logro, errores conceptuales, así como programar los mecanismos necesarios para superarlos. Todo ello en el marco de las reuniones de seguimiento del programa previamente programadas. Durante la aplicación del programa se pasarán el TEST DE CONTROL MEDIO y las distintas pruebas.

3. Evaluación final. Se realizó a través de la ejecución del TEST DE CONTROL FINAL.

Los maestros, maestras y la profesional del EOE implicada realizaron "entrevistas no estructuradas" periódicas (quincenales) con los alumnos durante los meses que duró la experiencia. En las primeras, se puso especial énfasis en la necesidad de mejorar la eficacia lectora tras los resultados en la primera evaluación. Posteriormente se informó a los alumnos sobre la aplicación de un Programa que les iba a ayudar a mejorar la eficacia lectora a través del entrenamiento respecto a la velocidad y la comprensión lectoras; en las siguientes, una vez iniciado el Programa, se pidió a los alumnos que se manifiesten libremente sobre su gusto por los distintos tipos de actividades de las distintas series, así como se les motivó para que realizaran la tarea con el máximo de atención y concentración. Conforme avanzaban las semanas, las entrevistas fueron cada vez más dinámicas y con una participación mayoritaria por parte de los alumnos implicados, hasta tal punto, que querían aumentar el número de series trabajadas diariamente. A través de estas entrevistas se les proporcionó un feedback positivo y se les informó de la mejora, estancamiento o incluso, en algún caso, del retroceso de los resultados 
obtenidos. Dos semanas antes de acabar la experimentación, la entrevista se centró en la opinión que el Programa merece a los sujetos implicados, tanto alumnos como maestros y profesional implicada del EOE.

\section{RESULTADOS}

\section{Relaciones entre los diferentes aspectos estudiados.}

Para establecer el grado de asociación entre las variables estudiadas (velocidad, comprensión y efectividad lectora) efectuamos análisis correlacionales con todas las puntuaciones directas obtenidas. Los resultados se recogen en la Tabla 4.

Tabla 4. Coeficientes de correlación de Pearson $(n=112)$

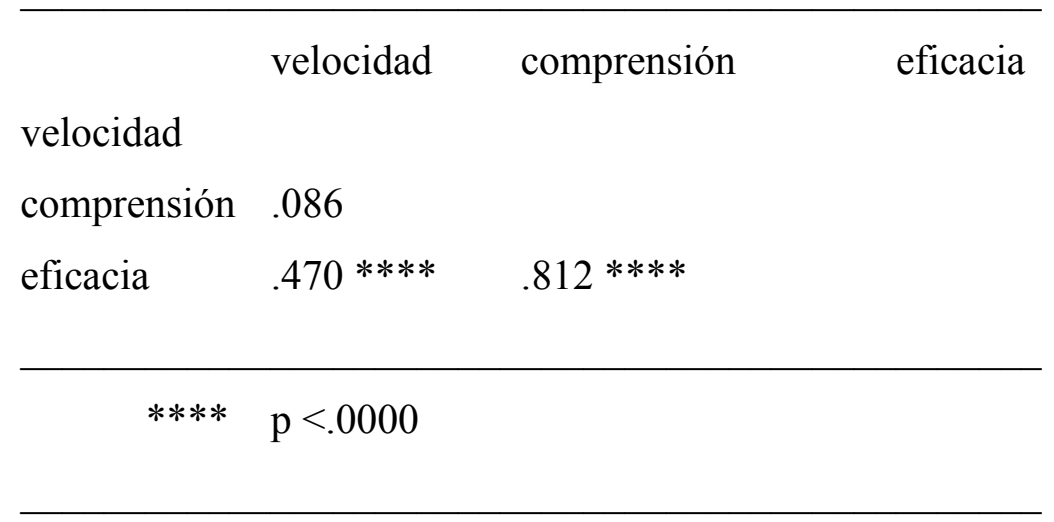

Como puede comprobarse, la comprensión y la velocidad están íntimamente asociadas a la eficacia lectora, aunque en el caso de la primera en mayor grado. Sin embargo, la velocidad y la comprensión lectora no muestran asociación significativa

\section{Efectos globales de la intervención efectuada.}

Para comprobar si los efectos cuantitativos de nuestra intervención eran adecuados, realizamos un análisis de diferencias de medias, entre las cuatro mediciones efectuadas. Los resultados se exponen en la Tabla 5. 
Tabla 5. Diferencias aparecidas (medias y desviación típica) entre las diferentes medidas en las variables estudiadas, a través de ANOVAs de medidas repetidas $(\mathrm{n}=27)$

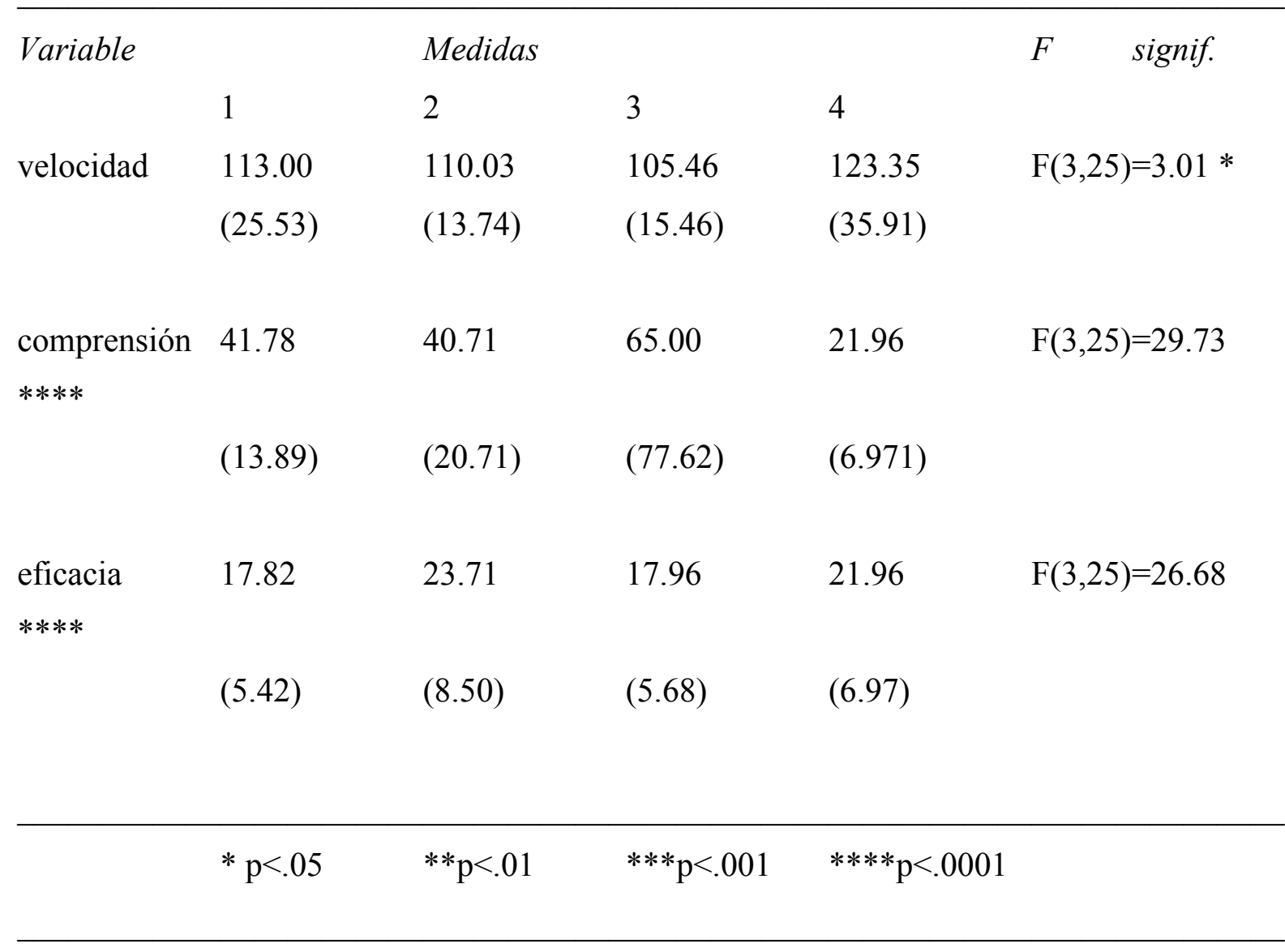

Los resultados encontrados muestran diferentes aspectos. En todos las variables analizadas se produce un efecto claro del momento (tratamiento) en la variabilidad de los resultados (velocidad, comprensión y efectividad). No obstante esto, los cambios no se producen siempre en la misma dirección. Así, mientras la velocidad sufre un incremento al final del período de entrenamiento, no ocurre así con la comprensión lectora, que tras un incremento baja al final del período de intervención. Finalmente, la eficacia lectora, como índice integrador de los dos anteriores se incrementa claramente desde el primer momento al último momento.

3. Efectos de la intervención por centros.

También quisimos comparar la evaluación diferenciada en cada centro. Los resultados se recogen en la Tabla 6. 
Tabla 6. Diferencias aparecidas (medias y desviación típica) entre las diferentes medidas en las variables estudiadas, a través de ANOVAs de medidas repetidas, para cada centro.

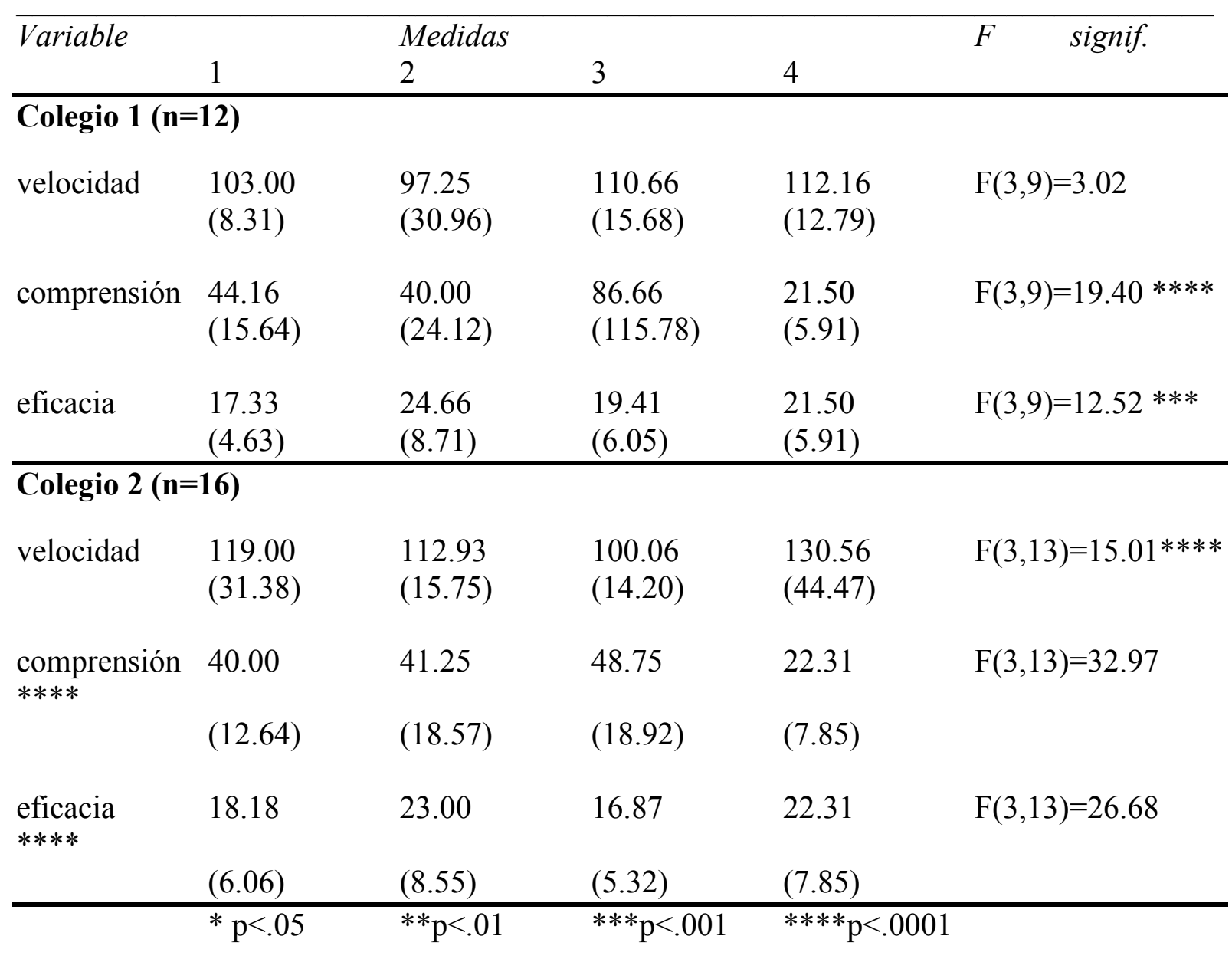

Los resultados denotan cierta significatividad, en la mejora de la velocidad y la comprensión. Obsérvese que el aumento de la velocidad mucho más significativo en el colegio 2. Por su parte, la comprensión disminuye sensiblemente en ambos casos. En el caso de la eficacia lectora, se produce un aumento significativo a lo largo de todo el proceso en ambos centros.

\section{DISCUSIÓN}

Al realizar la valoración final de la experiencia, consideramos necesaria hacerla desde dos puntos de vista: cuantitativo y cualitativo.

a) Desde el punto de vista cuantitativo podemos indicar que con respecto a la primera hipótesis (La velocidad y la comprensión lectora se perfeccionarán con el entrenamiento de 
habilidades lectoras), los datos obtenidos en la evaluación nos permiten afirmar que, esto es así con respecto a la velocidad, mientras que en el caso de la comprensión lectora, mejora significativamente en todos los momentos menos en el final. Dado que la velocidad siempre aumenta en la misma dirección, una posible explicación estaría en el hecho de que los alumnos están más atentos a la variable velocidad que a la comprensión. Esta idea nos lleva a constatar que, puesto que ambas variables están íntimamente relacionadas con la eficacia lectora, realmente, los alumnos no han llegado a autorregularse adecuadamente en el control y equilibrio de ambas variables.

En relación con la segunda hipótesis (La aplicación de un Programa determinado contribuirá a aumentar la eficacia lectora en los alumnos como producto de la mejora en la velocidad y la comprensión), hay que resaltar que la eficacia lectora ha aumentado significativamente en todos los casos.

Por otra parte, cuando analizamos los objetivos mensurables del Programa MATEL, podemos destacar que, con respecto al $1^{\circ}$ objetivo ("conseguir una velocidad de lectura superior a 200 palabras por minuto), ha sido sólo un 7,14\% de la muestra los que lo han conseguido, si bien es preciso destacar que un $71,42 \%$ de la población no superaba la velocidad de 120 palabras por minuto en la prueba control. Para conseguir alcanzar este objetivo, hubiera sido necesario aplicar el programa durante un periodo de tiempo mayor.

De igual manera, respecto al segundo objetivo (conseguir una comprensión lectora superior al 70\%"), sólo lo consigue un $21,42 \%$ de la muestra, pero igualmente es preciso destacar que un 53,57\% de la muestra no llegaba al 50\% de comprensión en la prueba control.

Respecto al tercero objetivo (obtener un buen índice de eficacia lectora) hay que afirmar que un $50 \%$ supera el $20 \%$ de eficacia lectora, siendo preciso destacar que al comienzo del Programa sólo un 28,57 alcanzaba el 20\% de eficacia lectora.

b)Desde el punto de vista cualitativo, podemos deducir ,de las sucesivas entrevistas con los alumnos y profesores implicados, que la valoración es plenamente satisfactoria por las siguientes razones:

- El Programa no cansa a los implicados, al revés conforme vamos avanzando en el tiempo va aumentado el entusiasmo ya que perciben los resultados 
positivos, por lo cual podemos afirmar que el Programa no causa saturación en los participantes.

- Los alumnos, tras un entrenamiento en el Programa y una correcta programación de las series por parte de los profesores y EOE implicados, son capaces de administrarse, casi de forma autónoma, la actividad diaria a trabajar.

- Obliga a los alumnos a mantener la atención en una actividad muy concreta durante un periodo de tiempo, con lo cual también mejora la capacidad de atención y concentración del sujeto.

- Es una ayuda muy importante para los profesores del ciclo ya que posibilita prestar una ayuda individual a cada alumno, durante muchas sesiones y de forma especializada para conseguir el desarrollo de determinadas destrezas lectoras.

- Después un curso de trabajo, los alumnos han puntuado la mayor parte de las series de trabajo con valores cercanos al 10.

Por último, es preciso destacar que, aunque no ha sido tratada de forma específica le escritura, hemos comprobado como ésta se ha beneficiado indirectamente. Estos resultados nos han sorprendido por no haber sido considerados previamente.

\section{REFERENCIAS}

Anula, J.J. (1995). La práctica del lenguaje integrado, donde motivación y comprensión van unidas. Comunicación: Lenguaje y Educación, 25, 51-69.

Calero, A. y Pérez, R. (1989).La madurez para la lectura.Enfoque cognitivo versus enfoque neuro-perceptivo. Investigación en la Escuela, 9, 25-33.

Foucambert, J. (1989). Cómo ser lector. Barcelona:Laila.

Grau, R. (1997). La lectura en la educación primaria. Aula de Innovación Educativa, 59, 3236.

Lacasa, P., Anula, J.J. y Martín, B. (1995). Leer y escribir:¿cómo lograrlo desde la perspectiva del lenguaje integrado?. Comunicación: Lenguaje y Educación, 25, 31-49.

López, J.L. (1992). PROLEC. MATEL Material para el entrenamiento lector. Valencia: José Luis López Rubio.

Lozano, L. (1990). Las dificultades de la lectura desde el procesamiento de la información. 
Comunicación: Lenguaje y Educación, 5, 105-116.

Martínez, J. (1994).La mediación en el proceso de aprendizaje. Madrid:Bruño.

Monereo, C. y Castelló, M.(1997). Las estrategias de aprendizaje. Barcelona: Edebé.

Quintanal, J. (Coor.) (1995). Para leer mejor. Madrid: Bruño

Sánchez, E. (1994). Estrategias de intervención en los problemas de lectura. En Marchesi A., Coll C. y Palacios J. (Comp.), Desarrollo psicológico y educación III (pp.139153).Madrid: Alianza psicología.

Sánchez, E.(1994).El aprendizaje de la lectura y sus problemas.En Marchesi A., Coll C. y Palacios J. (Comp.), Desarrollo psicológico y educación,III (pp. 139-153). Madrid:Alianza psicología.

Sánchez, E., Rueda, M. y Orrantia, J. (1998). Estrategias de intervención para la reeducación de niños con dificultades en el aprendizaje de la lectura y escritura. Comunicación: Lenguaje y Educación, 3, 101-111

Serra, C. y Oller, C.(1997). Estrategias lectoras y comprensión del texto en la enseñanza obligatoria. Aula de Innovación Educativa, 59, 24-27.

Solé, I. (1994). Estrategias de lectura. Barcelona: Paidós 